City University of New York (CUNY) CUNY Academic Works

\title{
Barrels XXXII Meeting Report: Whiskers in the Windy City
}

Giuseppe Cataldo

CUNY Queens College

Chia-Chien Chen

CUNY Queens College

Alicia C. Barrientos

CUNY Graduate Center

Joshua C. Brumberg

CUNY Queens College

\section{How does access to this work benefit you? Let us know!}

More information about this work at: https://academicworks.cuny.edu/qc_pubs/398

Discover additional works at: https://academicworks.cuny.edu

This work is made publicly available by the City University of New York (CUNY).

Contact: AcademicWorks@cuny.edu 


\title{
Barrels XXXII Meeting Report: Whiskers in the Windy City
}

\author{
Giuseppe Cataldo ${ }^{1}$, Chia-Chien Chen ${ }^{1}$, Alicia C. Barrientos ${ }^{2}$, Joshua C. Brumberg ${ }^{1,2,3 *}$
}

1. Department of Psychology, Queens College, CUNY

2. Behavioral and Cognitive Neuroscience program, The Graduate Center, CUNY

3. The Biology PhD Program, The Graduate Center, CUNY

Text pages: 31

Words in Abstract: 62

Tables: 0

Figures: 1

*Corresponding Author Joshua C. Brumberg

Department of Psychology

Queens College, CUNY

65-30 Kissena Boulevard

Flushing, NY 11367

Phone: 718-997-3541

Fax: 718-997-3257

Joshua.brumberg@qc.cuny.edu 


\begin{abstract}
:
The $32^{\text {nd }}$ Annual Barrels meeting was hosted at the Northwestern University Feinberg School of Medicine in Chicago, Illinois on October $17^{\text {th }}$ and $18^{\text {th }}, 2019$. The annual meeting brings together researchers who utilize the rodent whisker-to-barrel system as a means to understand cortical function and development. This year's meeting focused on social behaviors, development and cerebellar functions within the barrel system and beyond.
\end{abstract}


The annual Barrels meeting is the longest running satellite meeting to the annual Society for Neuroscience meeting. The $32^{\text {nd }}$ Barrels meeting was hosted within the Robert $\mathrm{H}$. Lurie Medical Research Center at the Feinberg School of Medicine located on the Northwestern University, Chicago Campus, and was held on October $17^{\text {th }}$ and $18^{\text {th }}, 2019$ (see Appendix 1 for the complete schedule). The Barrels meeting brings together researchers from around the world who use the rodent whisker-to-barrel cortex system as a model of among many things; cortical development, social behaviors, sensory processing, disease pathologies and motor actions. This year's meeting had several blocks of invited speakers, as well as short talks and a poster session, thus providing neuroscientists around the globe unique meeting opportunities to gather and discuss future scientific endeavors.

Jochen Staiger (Georg-August Universität Göttingen, Germany) introduced the first session of Barrels XXXII which focused on the, "Readjusting of cortical circuits during development".

Chinfei Chen (Harvard University) started off by presenting a talk entitled, "Wiring and Rewiring Information Lines in Sensory Thalamus". As with many central nervous system synapses, neuronal connections are initially imprecise but over time undergo refinement and can be reorganized with experience. This experience-dependent refinement of synaptic circuits is essential for normal neurological functioning. The visual system being one of the best understood sensory systems, serves as a model for studying sensory information processing and circuit development although how disruption of these circuits contributes to neurodevelopmental disorders is poorly understood. One particular example of this model for studying these processes is the retinogeniculate synapse, the connection between retinal 
ganglion cells and thalamocortical neurons in the visual thalamus a circuit where sensory information can be readily manipulated. In brief, visual information encoded in the retina is transmitted via retinal ganglion cells to thalamic relay neurons in the dorsal lateral geniculate nucleus (dLGN). Relay neurons then project to the primary visual cortex where higher order processing takes place. Using a combination of tools including patch clamp slice electrophysiology, in vitro single unit recordings and in vivo optical imaging methods, Dr. Chen showed that thalamic circuits demonstrate robust changes over development that shapes the nature of the information transmitted. Recent studies from her lab showed that this primary sensory pathway develops through the concurrent and interdependent remodeling of subcortical and cortical circuits in response to sensory experience. Deprivation during a critical period in development can disrupt the maturation of both the dLGN and the visual cortex leading to possible underlying factors for neurodevelopmental disorders.

Dan Feldman (University of California, Berkeley) gave the subsequent talk on using the "Somatosensory cortex as a model for understanding cortical circuit deficits in Autism". He started by asking, what is Autism? He then characterized the disorder by explaining that it most frequently includes sensory abnormalities which were tactile and auditory in nature, quite often displayed as a hypersensitivity in these modalities. Autism is highly genetic (approximately 50\%) involving about 100 genes which constitute the autism spectrum. Recent evidence suggests that there is nothing wrong with the intrinsic properties of the neurons themselves, but that the dysfunction lies within the circuit. Dr. Feldman employed the rodent whisker somatosensory cortex as a model system which allowed comparison of the same circuit across several different genetic strains of mice. These animals were used to test the excitation- 
inhibition (E-I) imbalance hypothesis which had been proposed by John Rubenstein and Michael Merzenich in 2003 who postulated that distinct genetic forms of autism share a common circuit basis in increased E-I ratio in the cerebral cortex. This results in circuit hyperexcitability, excess spiking and degraded information processing. Dr. Feldman used his understanding of feedforward excitation and inhibition in layers $2 / 3$ of primary somatosensory cortex (S1) to test this prevalent model of autism. His lab tested this hypothesis in four well established mouse models of autism (Fmr1-/y, Cntnap2-/-, 16p11.2del/+, and Tsc2+/-) and synaptic physiology experiments found that all four models had reduced inhibition along with a smaller decrease in excitation which yielded an increase in E-I conductance ratio. However, synaptic depolarization and whisker-evoked spiking in vivo were remarkably normal. They also showed that $\mathrm{E}$ and I conductance changes were quantitatively matched to produce stable, not increased synaptic depolarization, a sign of endogenous homeostasis. Thus, E-I ratio changes in these autism mouse models does not appear to drive network hyperexcitability but rather, represents a common homeostatic, compensatory response to circuit perturbation.

Next, after a short break, Julien Guy (Georg-August Universität Göttingen, Germany), presented on "Circuit imbalances and sensory processing in a model cortex without layers". A model of anomalous cortical development, the reeler mouse, is characterized by a loss of cortical lamination although some functional organization of the barrel field persists but in a distorted fashion. In this animal model, neurons are strewn across the cortical depth rather than formed into distinct layers with little known about the effects of such disturbed lamination on cortical function or sensory processing. Remarkably, it was found that there was no significant difference between wild type and reeler mice which showed a persistence of 
somatotopy even in the absence of cortical layers. More recently, this persistence was investigated further through a combination of in vitro patch clamp recordings and optogenetics and it was discovered that thalamocortical fibers that follow an atypical path from the ventral posteromedial nucleus to the cortex still manage to form synapses onto layer IV equivalent neurons. However, although a layer IV equivalent circuit is preserved in reeler mice, thalamocortical input to spiny stellate cells is weakened compared to controls. This weaker thalamic input may be due to hyperexcitability or loss of inhibition which was exhibited as a decrease in the connection probability and strength between fast spiking and spiny stellate neurons. More recently through the use of shadowpatching, two-photon guided intracellular recordings of identified neocortical pyramidal neurons which are direction selective were compared in wild type versus reeler mice in vivo. It was found that there was a reduced sensory input in reeler neocortical pyramidal neurons but those that fired were quite normal. Directional selectivity was also spared in these mice, exploratory behavior was normal despite ataxia and basic memory and sensation were preserved. Thus, although various small abnormalities were found, they may not be enough and or masked by cortical compensation maintaining homeostasis instead of showing phenotypic specific deficits. Therefore, it seems that lamination may not be necessary for cortical function.

Gavin Rumbaugh (Scripps Research Institute) ended the morning session with his work on the "Top-down control of tactile functions by the autism risk gene Syngap1". A common cause of sporadic intellectual disability is Syngap1 haploinsufficiency (i.e. heterozygous loss-offunction) in humans. It controls the trajectory of synapse and dendrite maturation as well as sculpting the neural network assembly in a circuit specific manner. Mutations disrupt 
developing pyramidal neurons, though it remains unclear if this process contributes to cognitive abnormalities. What are the circuit mechanisms driving the common phenotype associated with neurodevelopmental disorder (NDD) such as autism? With recent clinical trial failures, currently, there are no disease altering treatments for autism. It is believed that autism is caused by altered functional neural networks with the hypothesis that cortical dysfunction causes behavioral abnormalities. Fortunately, a substantial portion of NDD cases are monogenetic which allows the use of animal models where causal relationships can be established. In past studies, in vivo neurophysiological analysis in Syngap1 mice showed that neurons in the hippocampus and prefrontal cortex exhibited hyperexcitability whereas neurons in somatosensory cortex displayed hypoexcitability encoding information related to touch ultimately enabling different functions in different cell types. But how does Syngap1 haploinsufficiency impair systems-level tactile functions required to alter behavior? More recent data in a paradigm to study active touch behavior indicated that Syngap1 haploinsufficiency in forebrain excitatory neurons is both necessary and sufficient to produce touch driven behavioral deficits. Performance in a whisker-dependent learning task showed alteration even though free whisking appeared normal. Two-photon imaging data was also presented demonstrating that altered processing of touch-related signals in Syngap1 mice is autonomous to cortical excitatory neurons resulting in multiple forms of disrupted whisker touch dependent learning. Together, these data demonstrated that Syngap1 lines are a good model for NDDs as it disrupts sensory motor integration within the whisker system.

After a coffee break, the first session of short platform, 10 minute talks began which were moderated by Chris Moore (Brown University). 
The first speaker was Alicia Barrientos (Queens College and The Graduate Center, CUNY) who provided compelling data on "Exploring the relationship between microglia and the perineuronal net during early post-natal development of the primary somatosensory cortex". She began by describing the multiple roles microglia (MG) exhibit in the central nervous system such as immune and maintenance functions as well as how they play an important role in neural development by strengthening, modifying and eliminating synapses. Then, she went on to talk about perineuronal nets (PNN) and their impact on neuronal physiology within the barrel cortex where they prevent axons from synapsing and thereby restrict plasticity in adulthood. Using the mouse barrel cortex as a model system, she examined these two key components in neural development and plasticity by asking, how does sensory deprivation influence them? C57BL/6 mouse litters underwent trimming in addition to injections of saline, minocycline (MG inhibitor) and LPS (MG activator) until post-natal day 30. It was found that trimming dramatically altered MG morphology especially their processes which became withdrawn whereas MG density did not change. PNNs were also not affected by injections alone but in conjunction with trimming saw reduced density in thalamo-recipient layers. In conclusion, these studies suggest that sensory deprivation in the developing brain may leave it more vulnerable to factors that influence MG activity which may in turn be responsible for shaping PNNs.

Hui-Chen Lu (Indiana University) was the subsequent speaker focusing on "Receptor tyrosine kinases in establishing and maintaining whisker-related cortical circuits". Dr. Lu's work showed how dendritic patterns influence the receptive field and afferent connectivity of neurons which translates into cognitive function and behavior. She studied barrel cortex spiny 
stellate cells (bSCs) in layer IV at different times in development. Glutamatergic neurotransmission plays an important role in the development of sensory maps, it was previously found that glutamate transmission instructs cortical circuit formation but what are the underlying mechanisms? More recent data suggests that glutamate transmission influences dendritic patterning through two altered receptor tyrosine kinase families, nerve growth factor (NGF)/Tropomyosin receptor kinase A (TrkA) and fibroblast growth factors (FGFs) whose signaling is mediated through FGF receptors (FGFRs). But can abnormal neuronal activity alter this? Her hypothesis was that glutamate from different afferents are exerting their effects at different stages. They first engage NGF-TrkA to guide dendritic orientation, then engage FGFFGFR to establish dendritic segments to integrate synaptic inputs.

Greg Telian (University of California, Berkeley) presented next on "Context dependent sensorimotor processing during active sensation" which explored the relationship between sensorimotor cortex and a motor action. His hypothesis was that vibrissal primary sensory barrel cortex (vS1) and vibrissal primary motor cortex (vM1) modulate their own set of parameters during whisking. He compared vM1's role in sensory-motor processing between untrained mice (no goal context), and animals performing a whisker-dependent (goal context) discrimination task. Using extracellular recordings and optogenetic silencing of parvalbumin expressing GABAergic interneurons which exert robust and reliable inhibitory effects on the normal cortical network, the sensory representation of tactile stimuli in vM1 was investigated and whether vM1 activity is necessary for adaptive whisking strategies. It was found that vM1 represents tactile stimuli independent of vS1, presumably relying on direct thalamocortical input. In the no goal task mice, vM1 silencing had no effect on whisking kinematics, but 
conversely, had a dramatic impact when mice were engaged in the goal driven tactile discrimination task. He concluded that vS1 and vM1 are required but that the tactile environment VM1 can be encoded by VM1 independently of S1, and its control over whisker movements is context dependent, being specifically engaged during demanding sensory tasks where only if specific information is needed.

The last short talk was given by Chris Rodgers (Columbia University) on "The sensorimotor strategies and neuronal representations of whisker-based shape recognition in mice". He began by asking, how do you identify things around you? How do you choose a motor strategy? Humans and other animals such as rodents can identify objects by actively touching them in a coordinated exploratory motion using tactile sensation. Since mice identify objects by scanning them with their whiskers, Chris set out to reveal the sensorimotor strategies mice use to recognize objects, and how these strategies are executed by the flow of information across cortical layers. To study this, head-fixed mice were trained to distinguish between convex and concave shapes. What information does the mouse use to discriminate these two shapes? Discriminating between shapes is more complex than a simple pole detection task. For detection, mice sum up contacts across whiskers. For discrimination, they compare contacts and how whisker contacts change which mean something different between tasks. But what about networks in the brain? Contacts and spikes are contained in 'packets' defined by the whisk cycle. The superficial layers respond to contacts most strongly. Deep layer inhibitory cells are driven by whisker motion. This suggests a model in which superficial neurons integrate touch-evoked activity, while deep neurons integrate this touch information in the broader context of the task. Taken together, it is believed that the process of object recognition will be 
revealed by these studies providing further understanding into the fundamental cortical computations performed.

After a refreshing lunch, and an introduction by Randy Bruno (Columbia University), the first day's afternoon session commenced with a Plenary Lecturer by Hongkui Zeng (Allen Institute for Brain Science), who spoke about new data on categorization of neurons based on multiple techniques, including single-cell transcriptomics, spatial transcriptomics, single and multi-patching electrophysiology, full three-dimensional reconstructions of neuronal morphology, high throughput brain-wide connectivity mapping, and large-scale neuron activity imaging. New data focused on GABAergic neuron characterization that were previously thought to have many commonly shared features. However, her data demonstrated that there are some non-shared features depending on the brain region. For instance, hippocampal and neocortical GABAergic neurons are similar to other GABAergic neurons within the same area but differ across areas, whereas glutamatergic neurons largely remain distinct from one another. This type of open-access multi-level approach allows the neuroscientific community to understand the connection between neuronal morphology, physiological features, and transcriptomic signatures of each type of neuron in the brain. Building such knowledge provides a solid foundation for the thorough understanding of how the brain circuit performs computational functions.

Following a short break, the next session of short talks began under the moderation of Solange Brown (Johns Hopkins University).

Sebastian James (University of Sheffield, United Kingdom) presented computational data that axon branching plays a key role in the process of creating Dirichletiform pattern 
(which is based on a generalization of the Laplacian that can be defined on every measure space, without the need for mentioning partial derivatives) barrels with somatotopic order. He utilized reaction-diffusion style models, and showed that his model is incapable of generating patterns of cortical fields that are more intricate than the patterns already present in the molecular signals. However, only two guidance signaling gradients are needed for barrel patterning to form, when the model introduces competition between the branches of axons that originate from different barreloids. These results are such that perhaps as few as two chemical gradients can account for the observed barrel patterning.

Next Saikat Ray (Humboldt University and Charité Universitätsmedizin, Germany), followed by presenting research on seasonal effects on the primary somatosensory cortex of the tree shrew. Their brains become smaller and thinner during winter, and then recover back to their 'normal' size over the spring and summer months. Interestingly, only the cortex shrinks in winter and changes in layer 4 are responsible for most of the shrinkage. Specifically, its thickness is reduced by approximately $25 \%$. The number of neurons is also reduced by about $30 \%$ in layer 4 during the winter season. Using a two-photon imaging approach, they also found fewer touch suppressed neurons during winter, which implies that natural changes of season affects cortical processing.

Jiseok Lee (Carnegie Mellon University) next presented that layer 2/3 fosGFP neurons within the barrel cortex are more responsive to posterior medial nucleus of thalamus (Pom) inputs as compared to the Ventral posterior medial thalamus (VPM) inputs. Following a multiwhisker sensory association behavioral task, the fos-GFP neurons also increased their responses to Pom inputs. Two-photon in vivo imaging allowed for the identification of three functional 
groups based on response properties: Downregulated, Stable, and Upregulated in terms of brightness expression compared to baseline imaging. The trained group showed no change in the stability of L2/3 fosGFP neurons. However, fewer neurons express bright fosGFP after sensory deprivation.

Arindam Bhattacharjee (University of Tuebingen, Germany) concluded the first platform talks by presenting research on local vs. global coding schemes in human touch perception. By choosing the pulse's waveform frequency and amplitude, they varied local kinematics while being able to disentangle the global variables and intensity. All participants' performance was better at detecting stimuli that included local velocity cues vs those excluding it.

Following a coffee break, the second short platform talks were moderated by Alison Barth (Carnegie Mellon University).

Evan Harrell (University of Paris Sud, France) utilized a custom-built multi-whisker stimulator along with computational modeling to identify the features that are encoded in granular and infragranular barrel cortex neurons firing rates. They found half of the neurons code for velocity then focusing on feature encoding they found that slip-stick events are much more salient.

Yonatan Katz (Weizmann Institute of Science, Israel) looked at paired interhemispheric patch-clamp recordings in awake head-fixed mice to investigate firing correlations between cortical hemispheres. They then utilized chemogenetic and optogenetic tools to silence one hemisphere, to investigate if it affects the opposite hemisphere. They also investigated up versus down state's effects on how neurons in two sides of the hemisphere are correlated. 
They found axonal activity was negatively correlated with behavioral activity, which indicates transcallosal axons could be mediating the activity-dependence of correlations.

Keisuke Sehara (Humboldt University of Berlin, Germany) followed by presenting a new approach to rapidly track whisker motion in real time, by utilizing the Pixy camera-based color object tracking, and by modifying DeepLabCut software, a recently developed pattern recognition system to track high frequency whisking, with very low latency of $\sim 2 \mathrm{~ms}$.

Nadine Zweifel (Northwestern University) concluded the session by speaking about a new simulation framework that incorporates a realistic three-dimensional model to take account for the entirety of movement, and therefore would be more accurate in predicting the sensory input to the vibrissal system.

After a full day of invigorating talks, the meeting adjourned to the adjacent atrium and the annual poster session and buffet dinner was hosted.

The following day, Michael Brecht (Humboldt University, Berlin) chaired the morning series of 30-minute platform talks focused on the neocortical organization of social behaviors.

First to present was Michael Long (New York University, School of Medicine), who showcased his research examining sensorimotor transformations in the singing mouse (Scotinomys teguina) and speech perception and production in humans. S. teguina is a highly vocal neotropical rodent. A mouse will initiate a vocal exchange resulting in a countersinging response from another mouse, therefore making it a useful model to study the neural mechanisms that underlie natural social interactions, focusing on vocal exchanges. The orofacial motor cortex (OMC) was identified as a hot spot subserving these rapid sensorimotor transformations underscoring speech production. Intracortical stimulation of the OMC resulted 
in flexion of the vocal muscles. Lesion studies demonstrated that these mice are capable of singing by themselves even in the absence of OMC. Thus, the actual circuitry that allow these mice to sing are presumed to be sub cortical, arising from a central pattern generator. However, the OMC is a site of executive control, allowing the start and stop of the song, and also allowing for coordinated song.

The Long lab recorded from infragranular OMC cells while mice were singing or being played a song. When mice were singing, there were some cells that displayed an increase in spike frequency between song bouts. In some cases, there was very little activity before the onset of song and in other cases the activity is much higher. Thus, the firing activity of neurons in $\mathrm{OMC}$ during singing behavior was posited to be a sensory triggered motor preparation response. It was not just an auditory response.

In the second half of the talk, the focus shifted to human studies designed to uncover whether there are similar mechanisms involved in human speech. While listening to another person, one does not start planning one's response after the person has stopped talking, rather planning begins while listening. Broca's area (BA) is one region of interest. To date, there is no consensus on what BA does, but it has been implicated in linguistic processing, as it is located on the speech sensory motor cortex. To study speech planning, electrocorticography was utilized.

The critical information task was used to probe speech production. In this task, the patients were asked a series of questions, such as: The opposite of big is what word? As soon as the patient hears the critical word ("big"), they can start planning ("the opposite of big is small"). Researchers can then examine where the processing timeline begins while patients are 
engaged in this task. In neurosurgical patients, when the critical word is moved to the end of the sentence, the response is delayed, thus taking them longer to respond. While motor cortex comes online when a patient speaks, in BA, as soon as the critical information is presented and the patient starts planning activity increases and then reduces to below baseline when articulation happens. BA seems to be involved in the process before speech production; more specifically in speech planning, and not as involved in speech production itself. To summarize these findings, speech perception and production in mice is coordinated by OMC, whereas in humans, it is in BA.

Up next was Peggy Mason (University of Chicago) who gave a talk on the neurobiology of helping behavior in rodents. Helping is a rewarding behavior, and animals are empathically driven to help as they can "catch" the affect of the other animal. It is important to also note, however, the helping is resource-depleting and socially selective. There are also various types of bystanders. The Mason lab set up an arena where a rat was encased in an acrylic tube that could only be opened from outside. The tube had perforations to allow for visual, olfactory and tactile interactions with a free roaming rat. In this paradigm, food is not used to reinforce opening behavior. The rats are free to decide whether they will open the tube or not. The Mason lab demonstrated that free rats will open to free the trapped rats, and the behavior was not motivated by play, but rather by observing their companion was in distress thus motivating the free rat to help. Emotional regulation is required to help. In a separate study, the Mason lab demonstrated that pharmacological intervention blocking affect in rats resulted in failure to open the tube. 
One interesting feature of helping behavior is that there is a degree of social selectivity. Rats demonstrate empathy between familiars, and thus would open only to their cagemates. This raised the question whether rats would help strangers of its same species, or rats of a different species. The Mason lab found that rats do open to rats of the same species, and will open to rats of a different species if they had been exposed to them or cohabited with them. The key to enforcing helping behavior in rats is familiarity.

Next, the Mason lab tested several rat models of the bystander effect, a phenomenon termed and studied by John Darley. In humans, individuals in large groups would not act to help or alert others of an immediate or imminent danger. A diffusion of responsibility is thought to mediate the hesitation to help. In Mason lab's rat model, a subset of rats were sedated and utilized as "confederate" rats. Rats who were placed in the platform with confederate rats showed a greater latency to open the restrainer relative to control rats, which is a result comparable to human studies.

The Mason lab took this question further, asking whether the type of confederate makes a difference in a rat's latency to free the restrained rat. Thus, the lab tested whether using rats of a different strain as confederates accelerates or delays the latency to opening. They found that rats in the platform with confederates of a different strain perform like rats in the control condition (a platform in which there is no rat in the restrainer). Not only do Sprague Dawley rats in the platform with the "unfamiliar" Long Evans rats as confederates not take information from them-they also fail to help. In this experiment, a break is operationally defined as opening one day but not the next. In controls, the number of breaks are low relative 
to rats placed with unfamiliar confederates, which have higher breaks. Interestingly, when rats are placed with naïve bystanders, the number of openings increase relative to other groups. To conclude, the lab reports that when you have a confederate, the reinforcement of helping is antagonized, but naïve bystanders reduce the anxiety which may underlie the reason why rats will open earlier.

Following a short break, Keren Haroush (Stanford University) discussed her ongoing work examining social representation in the primate brain. She prefaced her work underscoring the importance of social prediction, a fundamental basis of human social interaction and cooperative behavior which allows us to assess another individual's state of mind, such as their thoughts and feelings. Imaging studies have implicated several brain regions, such as the cingulate cortex, that subserve social prediction, but little is known about how neural circuits or single neurons compute information related to social prediction. Defining how neural circuits within a brain region give rise to complex social interactions is a focus of the Haroush lab. To elucidate the neurobiological underpinnings of social prediction, the lab utilized an adapted Prisoner's Dilemma; a game theory approach. In this paradigm, a subject has the choice to cooperate or defect for each trial. It is important that the subject is able to anticipate the other's intention and probable decision on each trial, as their successful outcome is dependent on it. Being able to temporally parse between the monkey's choice, the time of the revelation of opponent's decision and reward delivery allowed the Haroush lab to tease apart the neuronal signals predicting the opponent's unknown decision compared to the monkey's own choice while separating the monkey's past responses, the social context, reward expectancy and the outcome. Using the approach described above, the Haroush lab showed similarities 
between how monkeys and humans play, as both use the same canonical strategies. The lab was also able to identify of neurons that distinguish between self and other in the dorsal anterior cingulate cortex (dACC) that signal the opponents choice before the decision is revealed; a class of neurons the lab calls "other-predictive" neurons. Disrupting activity of these neurons with microstimulation negatively influenced mutually beneficial interactions. These data lent support that the cingulate cortex plays a pivotal role in animals' behavior in social contexts.

Next, the Haroush lab designed a study where they show that in social contexts, inhaled oxytocin enhances cooperative behavior in monkeys, and these results are similar to that of humans. Interestingly enough, oxytocin administration decreased the predominance of otherpredictive neurons in the $\mathrm{AACC}$ and dampened the activity of the population prediction of the other's pending decision. These results further implicate the role of other-predictive neurons in cooperative behavior. The neuronal prediction correlated with the monkey's own decision following oxytocin administration, but no correlation was observed in monkeys who were administered saline. Thus, these data demonstrate that oxytocin influences interactive social behavior. These findings are important as they have implications for focused and specialized treatments for disorders where theory of mind is altered such as autism spectrum disorder and schizophrenia.

In summary, the Haroush lab identified a previously unknown population of neurons in the dACC known as other-predictive neurons, which are able to integrate and compute information related to social prediction and cooperative behavior, and oxytocin enhances cooperative behavior. 
The session concluded with moderator Michael Brecht (Humboldt University, Germany) taking the podium, who gave a talk entitled, "Blood is thicker than water," whose focus was on the neural mechanisms underlying kinship behavior. It is not well understood how organisms ranging from mammals to some insect species recognize kin, and how this recognition influences several social behaviors such as caregiving, attachment, mate selection and altruism. The Brecht lab utilized rats to examine kin preference, and found a developmental timeline where rat pups demonstrate a preference to their siblings at younger ages and then later develop non-sibling preference as they age. The question then was to identify what brain regions regulate or coordinate this behavior. A series of lesion-studies were performed. Kinpreference in young rats and non-preference in older rats was eliminated following damage to the lateral septum. To control for this, another cohort of rats underwent lesions to the cingulate cortex, and kinship-preference was not eliminated. Next, in vivo physiological experiments were conducted using Juxta-cellular and whole-cell patch clamp recordings from neurons within the lateral septum in awake-anesthetized rats. Rats were presented with odor cues and conspecific vocalizations from the rats' kin (mother and siblings) and non-kin (nonmother and non-siblings). They found preferential auditory and olfactory neuronal responses to individual social stimuli. Neurons in the dorsal lateral septum responded to non-kin odor, whereas neurons in the ventral lateral septum responded to kin odor. The Meyer lab is the first to report this ordered representation of response preferences regarding kinship, and thus have named it nepotopy, which could play a key role in studying differential behavior in mammals regarding kinship. To finalize, these data support the model that the lateral septum plays an instrumental role in organizing mammalian kinship behavior. 
After another fine lunch the fourth set of short talks commenced under the leadership of Garrett Stanley (Georgia Institute of Technology).

The first speaker was H. Philip Zeigler (Hunter College and The Graduate Center, CUNY) who provided data that texture discrimination required barrel patterning not just the presence of barrel cortex. Two strains of mice were utilized, a Prrxl1 knockout which lack patterning anywhere along the whisker-to-barrel cortex pathway and a barrelless mutant which lacks patterning only at the level of the barrel cortex. Both animals were able to perform a novel object recognition task, an olfactory discrimination task, and had similar whisker kinematics but neither were able to perform texture discrimination tasks which led to the conclusion that the barrel patterning was necessary for textural discrimination.

Next to present was Chia-Chien Chen (University of California, Santa Cruz), who utilizes a restraint stress paradigm that caused impaired performance on a texture-based novel object discrimination task. Using in vivo two photon imaging, he found that coupled with the decreased behavioral performance was an increase in the rate of dendritic spine elimination (spine formation remained the same) when compared to unstressed animals.

Immunohistochemical staining revealed that microglia appeared to be triggered as a result of the stress. Pharmacological blockade of microglia with minocycline returned the dendritic spine elimination rate to normal and performance on the novel texture discrimination task reverted to levels comparable to controls.

Edward Zagha (University of California, Riverside) presented a 'selective' detection task where the mouse had to use its whisker to identify the target from a distractor in response for a water reward. While the animals were performing the behavioral task, the brain was imaged 
using wide field $\mathrm{Ca}^{2+}$ imaging. In target trials, activity would propagate from S1 to M1 and then spread across a large expanse of the neocortex. When the distractor was present, there was activity in S1 but no further propagation to other neocortical structures suggesting that attention was selectively modulating a filter between S1 and M1.

The final speaker of this session was Arco Bast (Center of Advanced European Studies and Research, Bonn, Germany) who utilized the simulation environment NEURON to model a layer 5 thick tufted pyramidal cell. Using a two-parameter model that accounted for the timing and location of synaptic inputs, the output of the neuron could match receptive fields recorded in vivo.

Following a stimulating coffee break Mitra Hartmann (Northwestern University) opened up the final short talk session of Barrels XXXII.

First to speak was Yuri Vlasov (University of Illinois, Urbana-Champaign) who described a tactile virtual reality device, wherein head fixed animals would run on a Styrofoam ball and be 'directed' by glass walls that could be arbitrarily angled to create virtual mazes. Using electrophysiology, he was able to show that different areas display correlated neural activity across time.

John Barrett (Northwestern University) then demonstrated how agile mice could be with their thumbs in seed eating behaviors utilizing high speed videography. Analysis of the videos revealed the utilization of a bimanual precision grip and the correlation between eating and sniffing and the importance of constant regripping to optimal position seeds for eating.

The final speaker was Christian Ebbesen (Humboldt University, Berlin, Germany) who emphasized the importance of multisensory integration in social touch behaviors in rats. 
Recording in multiple sensory and motor cortical areas revealed that there were neurons who demonstrated gender preference, with a higher incidence observed in females. Further investigation suggested that the neurotransmitter oxytocin played an important role in social touch behaviors.

Following another coffee break, the meeting reconvened one final time for a session on Cerebellar Circuits in Behavior moderated by Dieter Jaeger (Emory University) who walked the attendees through the canonical circuitry of the cerebellum wherein Purkinje cells with their fan like dendritic trees receive inputs from extrinsic sources and then send their outputs via the deep cerebellar nuclei which in turn synapse onto cortical, thalamic and brainstem locations. It was noted that the cerebellum possesses a somatotopic map in its spino-cerebellum, but that it is not as fine grained as the cortical map. Classically, corrections in motor plans to changes in sensory or motor feedback required activation of the cerebellum.

The first invited speaker of the session was Richard Warren (Columbia University), who trained mice to run on a wheel wherein a bar was lowered and the animals had to clear the hurdle. Using high speed video analysis in the dark, the gait was analyzed and it was shown that when the whiskers were trimmed the leading leg would more often bang into the hurdle then when the whiskers were intact. If primary motor cortex was lesioned, the success rate in clearing the hurdle decreased to less than $50 \%$. In contrast, removing primary somataosensory cortex had no impact on the hurdle clearing behavior. Preliminary recordings from the cerebellum found evidence for the presence of overlapping sensory and motor signals.

Next to speak was Paul Chadderton (University of Bristol, United Kingdom), who focused on the nature of the whisker related signals in the cerebellum using primarily in vivo 
physiological techniques. Due to the presence of a full sensory representation in the cerebellum, its loss leads to deficits in coordination during locomotion. The whiskers are specifically represented in the lateral part of Crus 1. Recordings from Purkinje cells identified groups of cells that would increase their firing as well as groups of cells that decreased their firing rates in response to whisker stimulation. In general, the spiking patterns of Purkinje cells predicted the set point of the whisker, but did not encode the phase of whisking. Inactivation of primary motor cortex with the GABA agonist muscimol did not impact set point based on Purkinje cell firing rates. Whole cell recordings from Purkinje cells demonstrated that changes in the rates of excitatory post-synaptic currents were tuned for whisker position.

Finally, Clement Lena (Ecole Normale Superieure, Paris, France) closed the session and the meeting with a talk on how the cerebellum interacts with the whisker representations in primary motor and somatosensory cortices. It was emphasized that the cerebellum adjusts gain, reduces variance and adjusts the timing on motor actions. If the input from the primary somatosensory cortex to the cerebellum is blocked, animals will not perform a gap jumping task. Optogenetic studies revealed reciprocal connections between primary motor cortex and cerebellum. Studies that inactivated the deep cerebellar nuclei resulted in decreased firing rates in the neocortex in vivo and led to decreased performance on a novel object detection task. In sum, the three Cerebellar talks reinforced the crucial role that the Cerebellum has in constantly adapting to changes in the sensory periphery in order for the animal to be able to perform even the simplest tasks.

The meeting concluded with the determination to reconvene again despite the Covid19 pandemic this time online. 


\section{Figure Legend}

Figure 1. Barrels Attendees sitting in rapt attention during a talk in the Robert $\mathrm{H}$. Lurie Medical Research Center at the Feinberg School of Medicine located on the Northwestern University, Chicago Campus. Photo Credit: Jennifer Langland. 


\section{Acknowledgements:}

Thanks to the organizing committee; Solange Brown, Joshua C. Brumberg, Randy Bruno, Mitra Hartmann, Daniel O'Connor, Robert Sachdev, Gordon Shepherd Jr., and Jochen Staiger. The meeting was hosted by the Feinberg School of Medicine at Northwestern University and partially sponsored by Cambridge Electronic Design. Meeting assistance was provided by Drew Baughman.

\section{Disclosure of interest.}

The authors report no conflict of interest. 
Appendix I: BARRELS XXXII Program

Thursday, October 17

8:30 - 9:00 Coffee and continental breakfast

9:00 - 9:10 Welcome: Joshua Brumberg

The Graduate Center and Queens College, CUNY

"Readjusting cortical circuits during development"

9:10 - 9:25 Introduction/Overview: Jochen Staiger

Georg-August Universitaet Göttingen

9:25 - 9:55 Chinfei Chen

Harvard University

Wiring and Rewiring Information Lines in Sensory Thalamus

9:55 - 10:25 Dan Feldman, MW Antoine, T Langberg, P Schnepel

University of California, Berkeley

Somatosensory cortex as a model for understanding cortical circuit deficits in autism

10:25 - 10:30 Short break

10:30 - 11:00 Julien Guy

Georg-August Universitaet Göttingen

Circuit imbalances and sensory processing in a model cortex without layers

11:00 - 11:30 Gavin Rumbaugh

Scripps Research Institute

Top-down Control of Tactile Functions by the Autism Risk Gene Syngap1

11:30 - 11:45 Discussion

11:45 - 12:05 Coffee Break

Short Platform Talks 1 (10 min including questions)

Moderator: Chris Moore, Brown University

12:05 - 12:15 Alicia C. Barrientos, S. Mroziuk, A. Lahijani, J.C. Brumberg 
The Graduate Center, Queens College, CUNY

Exploring the relationship between microglia and the perineuronal net during early post-natal development of the primary somatosensory cortex

12:15 - 12:25 Jui-Yen Huang and Hui-Chen Lu,

Indiana University, Bloomington

Receptor tyrosine kinases in establishing and maintaining whisker-related cortical circuits

12:25 - 12:35 Greg Telian, J. Brown, H. Adesnik

University of California, Berkeley

Context dependent sensorimotor processing during active sensation

12:35 - 12:45 Chris Rodgers, R. Nogueira, B.C. Pil, E. Greeman, S. Fusi,

R.M. Bruno

Columbia University, New York

The sensorimotor strategies and neuronal representations of whisker based shape recognition in mice

$12: 45-2: 00$ Lunch

\section{Plenary Lecture}

2:00 - 2:50 Hongkui Zeng

Allen Institute for Brain Science

Integrated Cell Type Classification in the Mouse Cortex

2:50 - 3:00 Short break (no refreshments)

\section{Short Platform Talks 2 (10 min including questions)}

Moderator: Solange Brown, Johns Hopkins University

3:00 - 3:10 Sebastian S. James, J.M. Brooke, S.P. Wilson

The University of Sheffield 
The emergence of barrel and subbarrel patterning in-silico

3:10 - 3:20 Saikat Ray, M. Li, S. Mueller, P. Böhm-Sturm, M. Brecht, R. Naumann

Humboldt University and Charité Universitätsmedizin, Weizmann Institute of

Science, Chinese Academy of Sciences

Structural and functional seasonal plasticity in layer 4 of the adult somatosensory cortex

3:20 - 3:30 Jiseok Lee, A.L. Barth

Carnegie Mellon University

Sensory deprivation but not sensory training alters basal

fluctuations in cortical c-fos expressing ensembles

3:30 - 3:40 Arindam Bhattacharjee, C. Braun, C. Schwarz

University of Tuebingen, Germany

Human fingertip sensitivity to local coding variables of vibrotactile stimuli

3:40 - 4:00 Coffee Break

\section{Short Platform Talks 3 (10 min including questions)}

Moderator: Alison Barth, Carnegie Mellon University

4:00 - 4:10 Evan R. Harrell, M.A. Goldin, B. Bathellier and D.E. Shulz

Paris-Saclay Institute of Neuroscience (NeuroPSI), University of Paris Sud, France

A new set of higher order stimulus spaces reveals an elaborate slip-stick code in rat barrel cortex

4:10 - 4:20 Yonatan Katz, Y. Oran, M. Sokoletsky, K. Cohen, I. Lampl

Weizmann Institute of Science, Israel

The role of the corpus callosum in modulating interhemispheric synaptic correlations across behavioral states

4:20 - 4:30 Keisuke Sehara, P. Zimmer-Harwood, R. Bergmann, M.E. Larkum, R.N.S. Sachdev

Institute of Biology, Humboldt University of Berlin, Berlin, Germany

Tools for closed-loop, real-time tracking of mouse whisking behavior

4:30 - 4:40 N.O. Zweifel, M.J. Z. Hartmann 
Northwestern University, Department of Biomedical Engineering

A Three-Dimensional Dynamical Model of the Rat Vibrissal Array

4:40 - 9:00 Poster Session and Dinner

\section{Friday, October 18}

8:30 - 9:00 Coffee and continental breakfast

"Neocortical activity and the organization of social behaviors"

9:00 - 9:15 Introduction/Overview: Michael Brecht, Humboldt University, Berlin

9:15 - 9:45 Michael A. Long

New York University, School of Medicine

Neurobiology of Vocal Interactions

9:45 - 10:15 Peggy Mason

Department of Neurobiology, University of Chicago

The Neurobiology of Helping: Lessons from Order Rodentia

10:15 - 10:20 Short break

10:20 - 10:50 Keren Haroush

Stanford University

Social representation in the primate brain

10:50 -11:20 Michael Brecht and A. Clemens

Humboldt University, Bernstein Center for Computational Neuroscience Berlin

Blood is thicker than water

11:20 - 11:35 Discussion

11:35 - 1:00 Lunch

\section{Short Platform Talks 4 (10 min including questions)}

Moderator: Garrett Stanley, Georgia Tech 
1:00 - 1:10 Phil Zeigler, P. Feinstein, A. Resulaj, M.J.Z. Hartmann, M. Calvano, S. Bernal

Hunter College CUNY \& Northwestern University

Whisking-mediated texture discrimination (a) requires normal patterning along the trigeminal lemniscal pathway and (b) is barrel-pattern not barrel cortex dependent. The case of the barrelless mouse.

1:10 - 1:20 Chia-Chien Chen, J. Keating, Y. Zuo

University of California, Santa Cruz

Minocycline administration prevents stress-induced microglial morphological alterations and associated perceptual and synaptic defects.

1:20 - 1:30 K. Aruljothi, B. Zareian, K. Marrero, Z. Zhang, Edward Zagha

University of California, Riverside

Regulated Cortical Sensory-to-Motor Propagation during a Selective Detection Task

1:30 - 1:40 Arco Bast, R. Egger, M. Oberlaender

Center of Advanced European Studies and Research, Bonn Germany \& New York University

Synaptic properties can dominate cortical output computations

1:40 - 1:50 Short break

\section{Short Platform Talks 5 (10 min including questions)}

Moderator: Mitra Hartmann, Northwestern University

1:50 - 2:00 Ryan Loh, C. Graber, A. Schwing, Yurii Vlasov

University of Illinois at Urbana-Champaign

Dynamics of spiking correlations across S1 cortical layers in mice navigating in whisker-guided virtual reality

2:00 - 2:10 John M. Barrett, M.G.R. Tapies, G.M.G. Shepherd

Department of Physiology, Northwestern University

Manual dexterity of mice during food-handling involves the thumb and a set of fast basic movements

2:10 - 2:20 Christian L. Ebbesen, E. Bobrov, R.P. Rao, M. Brecht

Humboldt University, Neurocure Cluster for Excellence, Quest - BIH in Berlin \& NYU 
Highly structured, partner-sex and subject-sex-dependent cortical responses during social facial touch

2:20 - 2:45 Coffee Break

\section{"Cerebellar circuits in behavior"}

2:45 - 3:00 Introduction/Overview: Dieter Jaeger, Emory University

3:00 - 3:30 Richard Warren, Q. Zhang, J. Hoffman, E. Li, K. Hong, N. Sawtell

Columbia University

Whisker-Guided Sensorimotor Transformations On the Move

3:30 - 4:00 Paul Chadderton

University of Bristol

Organization of whisking-related signals at the input and output layers of cerebellar cortex

4:00 - 4:30 Clément Léna

Institut de Biologie de l'Ecole Normale Supérieure, Paris

Interactions of the cerebellum with whisker sensorimotor Cortices

4:30 - 4:45 Discussion

\section{ADJOURN}

